\title{
EFFECT OF SOIL TYPE, MOISTURE AND SAND COVER ON PUPATION DEPTH, SURVIVAL OF PUPAE AND ADULTS OF ZIZYPHUS FRUIT FLY, Carpomyia incompleta BECKER UNDER LABORATORY CONDITIONS
}

Risk, M. M. A. ; F. A. Abdel-Galil ; S. A. H. Temerak and Dalia Y. A. Darwish

Plant Protection Dept., Fac. Agric., Assiut University, Assiut, Egypt.

\begin{abstract}
The current study indicated that the most suitable soil type and moisture level for the emergence of Zizyphus fruit fly (ZFF), Carpomyia incompleta Becker (Diptera: Tephritidae) was the sandy soil containing 15\% water. It was found that the highest pupation depth was about $5 \mathrm{~cm}$. To avoid the harmful to the friendly soil fauna, it may be suggested that if the soil fumigation or gas producing materials must be applied, it should be penetrated into the soil not more than $10 \mathrm{~cm}$. The effect of soil depth on the survival of pupae and adults of ZFF was studied under laboratory conditions. The results indicated that, soil depth is negatively correlated with emergence of ZFF adults. The highest rates of fly's emergence (100-80\%) were observed at the lowest depths $(1-15 \mathrm{~cm})$ with no significant differences in the percentage of adult emergence. In fact, we have recorded that highest rates of emergence (100 and 95\%) were corresponded to 1 and $2 \mathrm{~cm}$ depth, respectively. The rates of emergence were gradually reduced when pupae placed on depth between 20 and $40 \mathrm{~cm}$. Rate of emergence at these depths was shown to be $70 \%$ at $20 \mathrm{~cm}$ depth and $45 \%$ at $40 \mathrm{~cm}$ depth. Depth of $50 \mathrm{~cm}$ had detrimental effect on emergence of adult fly, where the flies failed completely to emerge from the pupae $(0 \%)$ at this depth. The results showed also that depth of soil between 5 and $10 \mathrm{~cm}$ is the most preferable for emergence of ZFF adults.

Our results are evidence that soil depth had a significant impact on emergence and survival of adults of ZFF. Therefore, we suggest that soil depth should be considered as one of the most important abiotic factors in minimizing the pest's population.
\end{abstract}

Keywords: Zizyphus fruit fly, pupa, adult, moisture, soil depth.

\section{INTRODUCTION}

The Egyptian jujube, Zizyphus - spina - christi (L.) Desf., is an ancient prehistoric fruit, that usually known as Sidr and its edible fruit as Nabq. It is a common fruit grown in the worm subtropical regions, belongs to the genus Zizyphus of family Ghamnaceae. The economic importance of Nabq in Assiut Governorate comes after citrus, dates and pomegranate (Morsy, 1971; Abdel-Galial and Darwish, 1987 and Darwish-Dalia et al., 2012). Zizyphus trees are grown as a windbreak or border trees and as a popular food for a lot of people. This crop is highly sensitive to the infestation with Zizyphus fruit fly, C. incompleta (Farghal, et al., 1981). It is well known that the females of $C$. incompleta as the most of Tephritid flies deposit their eggs under the epidermis of Zizyphus fruits and after hatching of the eggs, the larvae feed exclusively on Zizyphus fruits. Infested fruits quickly rot and 
Risk, M. M. A. et al.

become inedible or drop to the ground. The full feed larvae ( $3^{\text {rd }}$ instar larvae) come out of fruits by boring an exit-hole to find a place to pupate in the soil (Kapatos, 1989; White Elsson-Harris, 1992). Pupation period lasts for 5-7 days and adult flies emerge and continue their life cycle, so this pest spends a part of its life cycle (pupal stage) inside the soil. Morsy, et al., (1981) reported that a proportion of $C$. incompleta pupae enters diapause by the end of April. Although, it is known that the pest pupate in the soil, there have been no accounts of the depth at which they pupate. One of the most effective items in IPM program for fruit flies is burying the fallen fruits in the soil. Therefore, knowledge of pupation depth of ZFF is necessary as it may affect pupation success, consequently emergence of adults due to difference in temperature, moisture, mortality factors such as predation, soil characteristics (Darwish-Dalia, et al., 2012) or efficiency control such as insecticidal soil drenches (Dimou, et al., 2003 and Renkema, et al., 2011). Thus, because of soil depth is an important abiotic factor affecting survival and developmental rates of ZFF, our simple question "Does the soil depth act as a direct mortality factor by the impact of soil pressure on pupae, or indirectly by preventing the emerged adults to make their way towards the soil surface?. The present question has still not been answered.

Therefore, the present study was carried out to estimate the soil depth at which the emergence of flies can be stopped, this may aid in developing alternative soil control methods, pupal sampling designs, assessment of natural mortality factors and management techniques in Zizyphus fruit and other fruit flies as well in Assiut Governorate.

\section{MATERIALS AND METHODS}

\section{Source of insects:}

In order to have a large number of pupae for this study, we have sampled infested Zizyphus fruits from different locations of Assiut Governorate. The fallen Zizyphus fruits under Zizyphus trees were collected in mesh pages twice a week during March and April, 2011 and transferred to the laboratory for examination.

Rearing cages:

The collected fruits were put in wire mesh cages $(20 \times 20 \times 20 \mathrm{~cm})$ with accurate holes. These cages were then suspended inside wooden crates each lined with two opposite aspects of wire mish. The upper aspect was made of glass. The frontal and rear aspects were made of wood with an opening of about $15 \mathrm{~cm}$ in the middle of the frontal aspect. This opening was provided with a black cloth sleeve. The base of the fund was also made of wood. Prior to experimentation, sand samples were washed, air dried, sifted through a $2-\mathrm{mm}$ mish metal sieve and dried in an oven for $6 \mathrm{~h}$ at $80^{\circ} \mathrm{C}$ to kill arthropods. Layers of sand of $2 \mathrm{~cm}$ for burying of the larvae during pupation were placed on the bottom of wooden cages. After that the pupae recuperated by sieving the sand. 


\section{Experimental Protocol:}

To get pupae free from parasites for this trial, the stock of pupae were placed in a black jar connect with transparent plastic vial (plate 1). This unit was observed daily tell the emergence of parasite. The parasite was then attracted to the light in the transparent vial. Emerged parasites were daily released a way from the plastic vials. Adults from healthy pupae were usually emerged two days after the parasites emergence. After confirmation of complete emergence of the parasites, the remaining healthy pupae were selected and immediately used in the experiment.
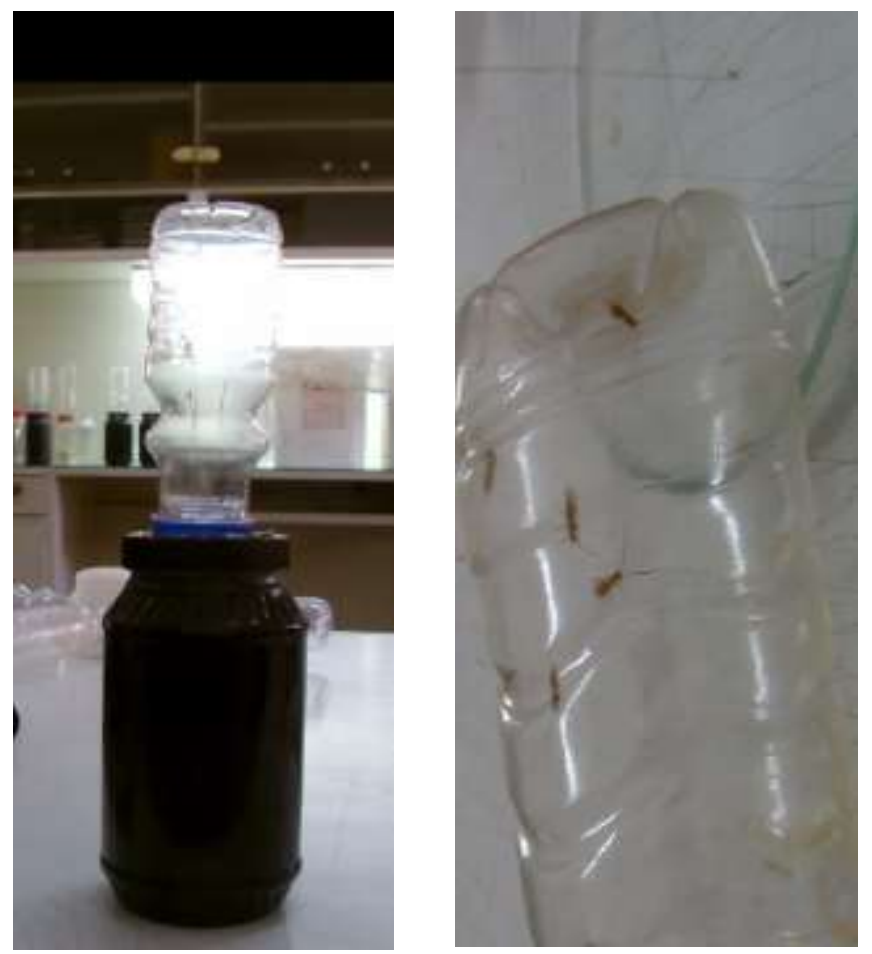

Plate 1: Parasite separator at the left side and emerged parasite attracted to the light at the right side.

\section{Effect of soil type and soil moisture on pupation depth:}

Three types of soil were prepared:

1-Clay: brought in from the farm

2-Sandy: a pure sand

3-A mixture of clay and sand (1:1) by volume

Five degrees of moisture, $0,5,10,15$, and $20 \%$ were prepared of soil and water volume by volume.

Plastic tubes $(27 \mathrm{~cm}$ length and one inch in diameter) were divided longitudinally into two pieces, collected together with rubber band, closed from one end by plastic sheet and packed by the wetted soil. Two $\mathrm{cm}$ at the top of the unit were left without soil. Ten full grown larvae were placed on the 
top of the soil, and covered with plastic sheet. Three replicates were prepared for each treatment. After 48 hours, the pupation depth was measured (Plate2).

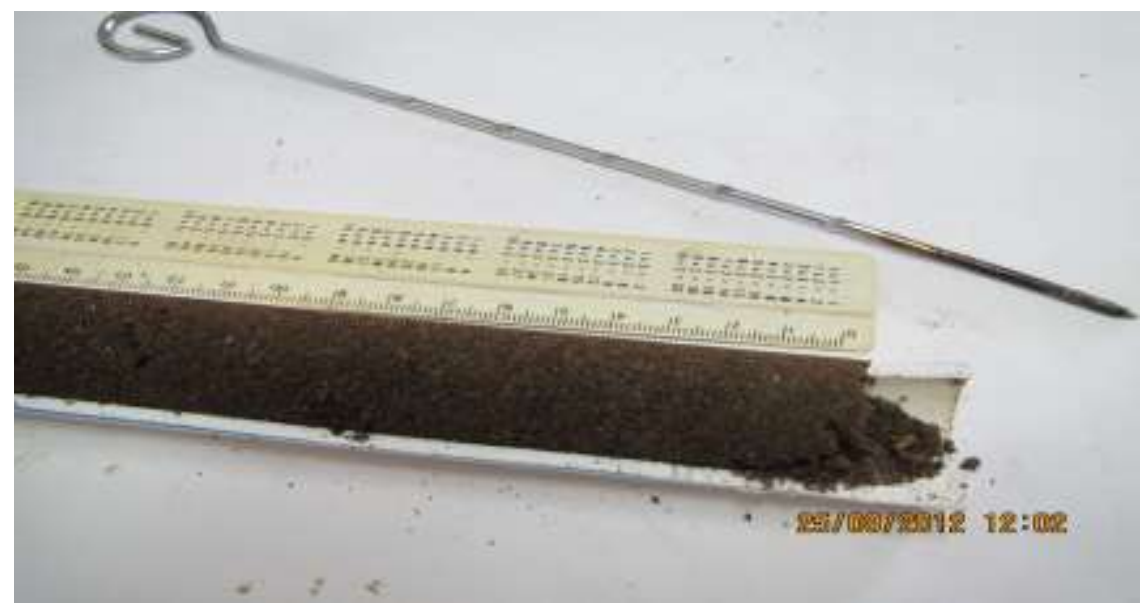

Plate 2: A method for evaluating the pupation depth of Zizyphus fruit fly

\section{Effect of sand cover weight on the emergence of Zizyphus fruit fly}

Ten depths $(3.5,5.5,7.5,12.5,17.5,22.5,27.5,32.5,42.5$ and 52.5 $\mathrm{cm}$ ) were tested to determine the effect of soil depth on emergence of Zizyphus fruit fly. Plastic tubes with a diameter of 2.4" were cut into pieces of different lengths corresponding with the studied depths. Each length was replicated four times. Metal covers were installed on the basal ends of tube pieces. The top ends of the tube pieces were provided with plastic containers to receive the emerged adult flies (plate 3 ).
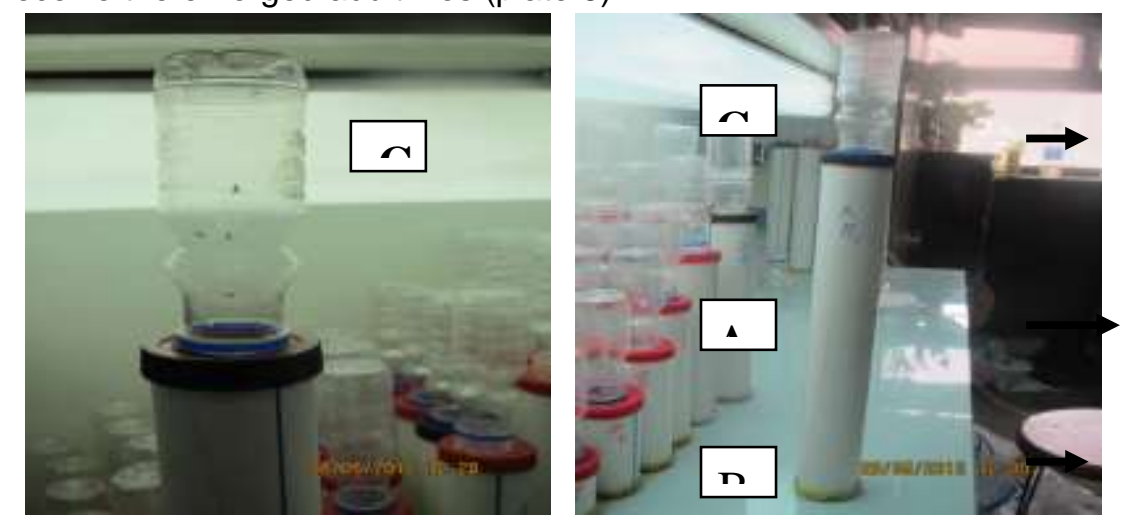

Plate 3: A tool used to determine the effect of soil depth on the emergence of Zizyphus fruit fly. A, Plastic tube $6 \mathrm{~cm}$ in diameter and different lengths ranged from 3.5 to $52.5 \mathrm{~cm}$, B lower stopper carrying the tested pupae, and $C$ upper stopper attached to a transparent plastic vial showing the emerged flies. 
Eighty healthy pupae (20 pupae/replicate) were placed on the bottom of tubes. The tube pieces were then filled with sand. The experiment was conducted in laboratory conditions at a temperature of $27 \pm 2^{\circ} \mathrm{C}$ and a relative humidity of $65 \pm 5 \%$. From the first emergence, the number of adults was daily counted in order to assess the rate of emergence at each soil depth. Heights of the sand inside the plastic tube pieces were converted into so called weight of sand $/ \mathrm{cm}^{2}$ according to the following formula:

Wt. sand $/ \mathrm{cm}^{2}=\pi(\mathrm{D} / 2)^{2} \times \mathrm{H}$

Where:

$\Pi=$ a constant $=3.14$

$\mathrm{D}=$ Tube diameter $(\mathrm{cm})$

$\mathrm{H}=$ Height of sand inside the tube piece

\section{RESULTS AND DISCUSSION}

\section{Effect of soil type and soil moisture on pupation depth of ZFF:}

The two-way analysis of variance of data (Table 1) indicated that the most suitable soil and moisture for the emergence of ZFF was the sandy soil containing $15 \%$ water.

Regardless of moisture content, the pupation depth was 1.68 and $1.56 \mathrm{~cm}$ in sandy and sand clay soils without significant difference. Beside this, the statistical analysis proved insignificant difference between moisture levels of $0,5,10$ and $20 \%$.

From the previous results it was found that the highest pupation depth was about $5 \mathrm{~cm}$. To avoid the harmful to the friendly soil fauna, it may be suggested that if the soil fumigation or gas producing materials should be applied, it must be penetrated into the soil not more than $10 \mathrm{~cm}$.

Table 1: Effect of soil type and soil moisture on pupation depth of ZFF

\begin{tabular}{|c|c|c|c|c|c|c|c|}
\hline \multirow[b]{2}{*}{ Soil type } & \multicolumn{7}{|c|}{ Moisture percentage } \\
\hline & $0 \%$ & $5 \%$ & $10 \%$ & $15 \%$ & $20 \%$ & \multicolumn{2}{|c|}{ Average } \\
\hline Clay & 2.2 & 1.9 & 1.5 & 1.5 & 1.4 & 1.682 & b \\
\hline Sandy & 1.9 & 1.5 & 1.2 & 16.2 & 1.7 & 4.492 & $\mathbf{a}$ \\
\hline Sand/Clay mixture 1:1 & 1.5 & 1.6 & 1.3 & 1.6 & 1.9 & 1.556 & b \\
\hline \multirow{2}{*}{ Average } & 1.8 & 1.7 & 1.3 & 6.4 & 1.7 & & \\
\hline & b & b & b & $\mathbf{a}$ & b & & \\
\hline LSD For Soil Type (5\%) & 2.5 & & & & & & \\
\hline LSD for Soil Moisture (5\%) & 0.32 & & & & & & \\
\hline LSD for Interaction (5\%) & 0.55 & & & & & & \\
\hline
\end{tabular}

\section{Effect of sand cover weight on the emergence of ZFF}

As shown in Table 2 the soil depth is negatively correlated with emergence of Zizyphus fruit fly adults. The highest rates of fly's emergence (100 and $80 \%)$ were observed at the lowest depths $(1-15 \mathrm{~cm})$ with no significant differences in the percentage of adult emergence. Rigamonti (2004) noticed that the pupae of Ceratitis capitata are formed at a depth inferior to $10 \mathrm{~cm}$ and $90 \%$ of the obtained pupae were concentrated in the five upper centimeters. Also, Ali et al., (2007) reported that the higher rate of 
Risk, M. M. A. et al.

emergence of $C$. capitata (69.3\%) was observed at $2 \mathrm{~cm}$ depth. Similar results were reported by Al-Zaghal et al., (1987) who observed maximum pupae of Dacus oleae at depth of $2.5-7.5 \mathrm{~cm}$ in soil under olive trees in Jordan. Malem and Giliomme (1968) observed maximum pupation of Dacus ciliatus in dry sandy soils at depth of 1 to 2 inches. The results of the abovementioned investigators are in close agreement with our results, we have in fact recorded that the higher rates of emergence (100 and 95\%) were corresponded to 1 to $2 \mathrm{~cm}$ depth, respectively. The data in Table (2) also show that rates of emergence gradually reduced when pupae placed at depths between 20 and $40 \mathrm{~cm}$. The rate of emergence at these depths was shown to be $70 \%$ at $20 \mathrm{~cm}$ depth and $45 \%$ at $40 \mathrm{~cm}$ depth. Depth of $50 \mathrm{~cm}$ had detrimental effect on the emergence of adult fly, where the flies failed completely to emerge from the pupae $(0 \%)$ at this depth.

Table (2): Effect of soil depth on the emergence of Zizyphus fruit fly adults.

\begin{tabular}{|l|c|c|c|}
\hline $\begin{array}{c}\text { Soil depth } \\
(\mathbf{c m})\end{array}$ & $\begin{array}{c}\text { Wt. soil } \\
\left(\mathbf{g m} / \mathbf{c m}^{\mathbf{2}}\right)\end{array}$ & $\begin{array}{c}\text { No. emerged flies } \\
\text { (mean) }\end{array}$ & $\begin{array}{c}\text { Emerged flies } \\
\text { (\%) }\end{array}$ \\
\hline 1 & 1.49 & 20 a & 100 \\
\hline 2.5 & 3.73 & 19 a & 95 \\
\hline 5 & 7.45 & 19 a & 95 \\
\hline 10 & 14.90 & 17 abc & 85 \\
\hline 15 & 22.35 & 16 abc & 80 \\
\hline 20 & 29.80 & 14 bcd & 70 \\
\hline 25 & 37.25 & $13 \mathrm{cde}$ & 65 \\
\hline 30 & 44.70 & 10 de & 50 \\
\hline 40 & 59.60 & 9 e & 45 \\
\hline 50 & 74.50 & $0 \mathrm{f}$ & 0 \\
\hline
\end{tabular}

L.S.D = 2.038

Means in the column followed by the same letter are not significantly different at 0.05 level of probability.

The present results suggest that emergence of ZFF in relation to soil depth could be classified into 3 different groups on the base of percent of emergence. The first had the highest emergence rates (80-100\%) with no significant differences among them; the second had moderate emergence rates $(45-70 \%)$ with no considerable differences in percent of adult emergence. The third group had no emergence $(0 \%)$ at all. Statistical analysis also indicated significant differences between first (1-15 cm depth) and second (20-40 cm depth) groups and between third (50 cm depth) and both first and second groups in respect to emergence percent.

Our results could be interpreted on the base that weights of soil between 1.49 and $22.35 \mathrm{gm} / \mathrm{cm}^{2}$ corresponding to depths of $1-15 \mathrm{~cm}$ were much lighter on the pupae so that adults emerged from these pupae were able to penetrate the soil very easily, while in case of pupae placed at $50 \mathrm{~cm}$ depth, the weight of soil on the pupae $\left(74.5 \mathrm{gm} / \mathrm{cm}^{2}\right)$ was too heavy so that the flies entirely failed to emerge from pupae. The present results clearly show that depth of soil between 5 and $10 \mathrm{~cm}$ is the most preferable for emerging of Zizyphus fruit fly adults from the pupal stage. 
It may be concluded that, results of the present investigation can be used in developing no-chemical control measures and designing efficient sampling technique for the insect in the ground. Based on our results we suggest that soil depth should be considered as a one of the important abiotic factor in minimizing the pest's population.

From the previous results it may also be suggested that when burying the infested fallen fruits in the soil, the later must be covered with a layer of clay or clay sand soil not less than $50 \mathrm{~cm}$.

\section{REFERENCES}

Abdel-Galil, F. A. and Y. A. Darwish (1987): Soil fauna of Zizyphus fruit trees in Assiut area. Assiut J. Agric. Sci., 18 (2): 61-72.

Ali A. D.; N. Soltani.; A. Kellouche and F. Mazouzi (2007): Effects of soil texture and the burying depth of the larvae on some biological parameters of Ceratitis capitata (Diptera: Trypetidae). African J. Agric. Res., 2 (3): 105-111.

Al-Zaghal, K.; T. Mustafa and K. A. Zaghal (1987): Studies on the pupation of the olive fruit fly Dacus oleae Gmel. (Diptera: Tephritidae) in Jordan. J. App. Entomol., 103: 452-456.

Darwish, Dalia Y. A.; F. A. Abdel-Galil; M. M. A. Risk; and S. A. H. Temerak (2012): Multi-correlation analysis between some vital aspects of Zizyphus fruit fly, Carpomyia incopmleta Becker, (Diptera: Tephritidae) and soil characteristics. Assiut J. Agric. Sci., 43(2): 156-163 (Special Issue) (The $6^{\text {th }}$ Conference of Young Scientists, Fac. Agric., Assiut Univ., May, 13, Assiut, Egypt.

Dimou, I., C. Koutsikopoulos; A. P. Economopoulos and J. Lykakis (2003): Depth of pupation of the wild olive fruit fly Bactrocera (Dacus) oleae (Gmel.) (Diptera: Tephritidae), as affected by soil abiotic factors. J. Appl. Entomol., 127: 12-17.

Farghal, A. I.; M. A. A. Morsy and F. A. Abdel-Galil (1981): Effect of soil treated with certain insecticides Jha's and chitin inhibitor on the Zizyphus fruit fly and its parasite. $1^{\text {st }}$ Inter. Cong. Soil Poll. Part II: 135146.

Kapatos, E. T. (1989): Immature stages, pp. 185-191. In A. S. Robinson and G. Hooper [eds.], Fruit flies: their biology, natural enemies and control, vol. 3B. Elsevier, Amsterdam.

Malem, E. M. and J. H. Giliomee (1968): Aspects of the bionomics of Dacus ciliatus Loew. J. Entomol. Soc. South Africa, 31: 373-389.

Morsy, M. A. A. (1971): Studies on certain fruit pests in Upper Egypt. M. Sc. Thesis, Fac. Agric. Assiut Univ. Egypt.

Morsy, M. A. A.; F. A. Abdel-Galil and S. A. Temerak (1981): Thermal regulation of pupal diapause in Zizyphus fruit fly Carpomyia incompleta Becker (Diptera: Trypetidae). $4^{\text {th }}$ Arab Pesticide Conf. Tanta Univ., III A: 179-186. 
Risk, M. M. A. et al.

Renkema, J. M.; G. C. Cutler; D. H. Lynch; K. Mackenzie and S. J. Walde (2011): Mulch type and moisture level affect pupation depth of Rhagoletis mendax Curran (Diptera: Tephritidae) in the laboratory. J. Pest Sci., 84 (3): 281-287.

Rigamonti, I. E. (2004): Contribution to the knowledge of Ceratitis capitata Wied. (Diptera: Tephritidae) in Northern Italy. II. Overwintering in Lombardy. Bull. Zool. Agr. Bachic. II, 36 (1): 101-111.

White, I. M. and M. M. Elson-Harris (1992): Fruit flies of economic significance: their identification and bionomics. CAB, Wallingford, U.K.

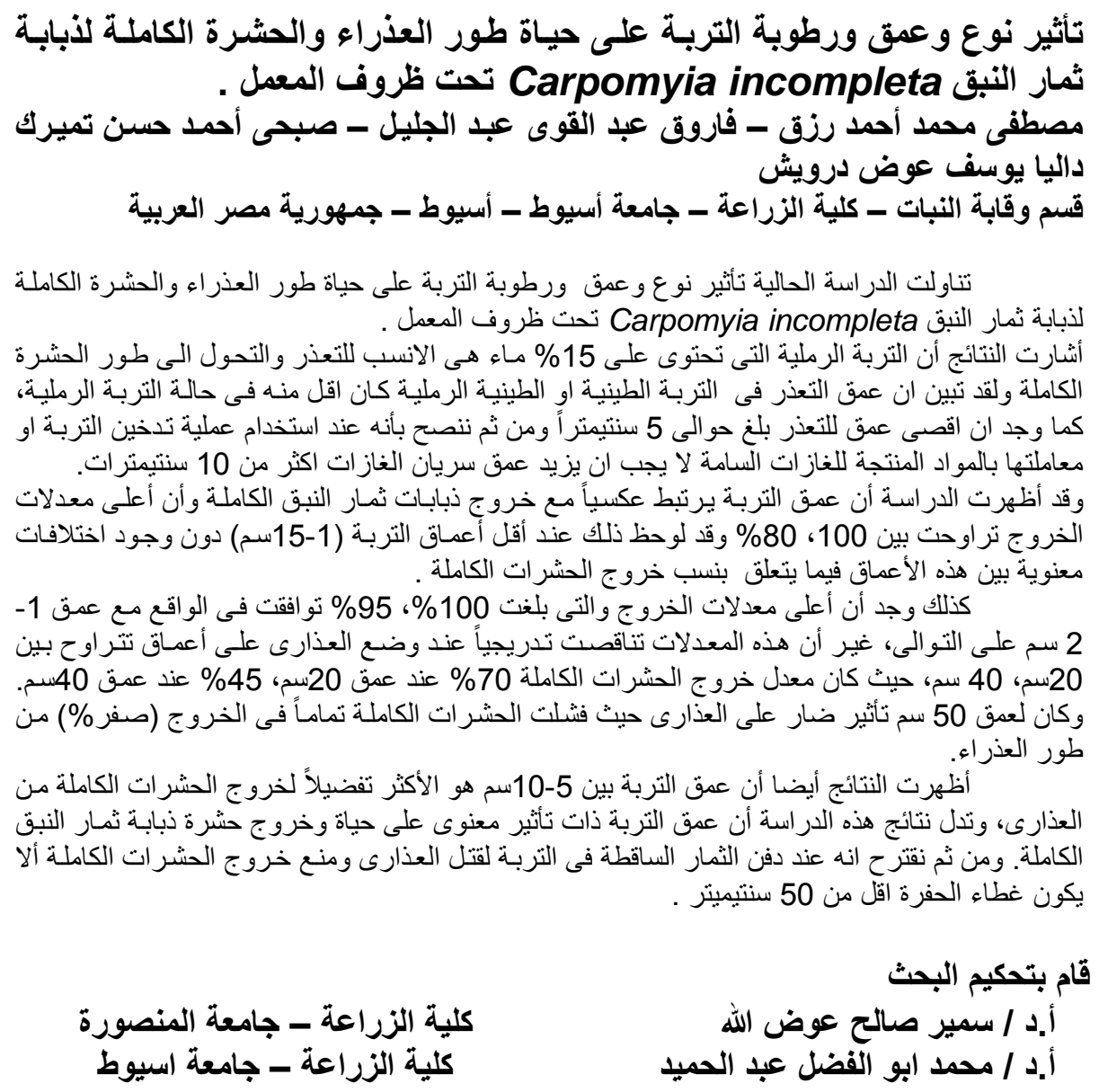

